\title{
To Understand the Four Cosmological Interactions
}

\author{
U. V. S. Ses havatharam ${ }^{1, *}$, S. Laks hminarayana ${ }^{2}$ \\ ${ }^{1}$ Honorary faculty, I-SERVE, Alakapuri, Hyderabad, 35, AP, India \\ ${ }^{2}$ Dept. of Nuclear Physics, Andhra University, Visakhapatnam, 03, AP, India
}

\begin{abstract}
Within the expanding cosmic Hubble volume, Hubble length can be considered as the gravitational or electro magnetic interaction range. Product of 'Hubble volume' and 'cosmic critical density' can be called as the "Hubble mass". The three key assumptions are: 1) within the Hubble volume, each and every point in free space is influenced by the Hubble mass, 2) 'molar electron mass' can be considered as the rest mass of a new heavy charged elementary particle and 3) atomic gravitational constant seems to be Avogadro number times the classical gravitational constant. This is a new approach and may be given a chance in understanding the four fundamental cosmo logical interactions.
\end{abstract}

Keywo rds Hubble Volume, Critical Density, Hubble Mass, Coulo mb Mass, Molar Electron Mass, Atomic Gravitational Constant and the CMBR Temperature

\section{Introduction}

"Hubble volume" can be considered as a key tool in cosmology and unification. In this paper an attempt is made to understand the basic unified concepts of the four fundamental cosmological interactions. This is a new approach and particle physics and cosmology can be studied in a cohesive mode.

\subsection{Basic Assumptions in Particle Cosmology}

With reference to the Mach's principle[1-6] and the Hubble volume, if "Hubble mass" is the product of cosmic critical density and the Hubble volume[7-9], then it can be assumed that,

1) With in the Hubble volume, each and every point in free space is influenced by the Hubble mass.

2) With in the Hubble volume, the Hubble mass plays a vital role in understanding the properties of electromagnetic and nuclear interactions.

3) Within the Hubble volume, Hubble mass plays a key role in understanding the geometry of the universe.

With reference to the Avogadro number[10] and from unification point of view, the utmost fundamental question is: How to understand the origin of "mass" of elementary particles? In this connection it can be assumed that,

1) "Molar electron mass" can be considered as the rest mass of a new heavy charged elementary particle.

2) Atomic gravitational constant is Avogadro number times the classical gravitational constant.

* Corresponding author:

seshavatharam.uvs@gmail.com (U. V. S. Seshavatharam)

Published online at http://journal.sapub.org/astronomy

Copyright (C) 2012 Scientific \& Academic Publishing. All Rights Reserved

\section{Key Concepts in Particle Cosmology}

Concept-1: In atomic and nuclear physics, atomic gravitational constant $\left(G_{A}\right)$ is Avogadro number times the classical gravitational constant $\left(G_{C}\right)$.

$$
G_{A} \cong N G_{C}
$$

This idea may come under the subject classification of "strong gravity" and is not in the main stream physics. K.P. Sinha, C. Sivaram, Abdus Salam, E. Recami and colleagues developed the subject in a unified gravitational approach [11-15]. It is reasonable to say that - since the atomic gravitational constant is $N$ times the classical gravitational constant, atoms are themselves arranged in a systematic manner and generate the "gram mole".

Concept -2: The key conceptual link that connects the gravitational and non-gravitational forces is - the classical force limit

$$
F_{C} \cong\left(\frac{c^{4}}{G_{C}}\right) \cong 1.21026 \times 10^{44} \text { newton }
$$

It can be considered as the upper limit of the string tension. In its inverse form it appears in Einstein's theory of gravitation[6] as $\frac{8 \pi G_{C}}{c^{4}}$. It has multiple applications in Black hole physics and Planck scale physics[16]. It has to be measured either from the experiments or from the cosmic and astronomical observations.

Concept -3: Ratio of 'classical force limit $\left(F_{C}\right)$ ' and ' weak force magnitude $\left(F_{W}\right)$ ' is $N^{2}$ where $N$ is a large number close to the Avogadro number.

$$
\frac{F_{C}}{F_{W}} \cong N^{2} \cong \frac{\text { Upper limit of classical force }}{\text { nuclear weak force magnitude }}
$$


Thus the proposed weak force magnitude is $F_{W} \cong \frac{c^{4}}{N^{2} G_{C}} \cong 3.33715 \times 10^{-4}$ newton. Considering this $F_{W}$, Higgs fermion and boson masses can be fitted. In this connection please see our earlier published papers[17-21] and application-9 of this paper.

Concept-4: In the expanding cosmic Hubble volume, $R_{0} \cong\left(c / H_{0}\right)$, can be considered as the gravitational or electro magnetic interaction range.

Concept-5: In the expanding cosmic Hubble volume, characteristic cosmic Hubble mass is the product of the cosmic critical density and the Hubble volume. If the critical density is $\rho_{c} \cong\left(3 H_{0}^{2} / 8 \pi G\right)$ and characteristic Hubble radius is $R_{0} \cong\left(c / H_{0}\right)$, mass of the cosmic Hubble volume is

$$
M_{0} \cong \frac{c^{3}}{2 G H_{0}}
$$

Concept-6: There exists a charged heavy massive elementary particle $M_{X}$ in such a way that, inverse of the fine structure ratio is equal to the natural logarithm of the sum of number of positively and negatively charged $M_{X}$ in the Hubble volume. If the number of positively charged particles is $\left(\frac{M_{0}}{M_{X}}\right)$ and the number of negatively charged particles is also $\left(\frac{M_{0}}{M_{X}}\right)$ then

$$
\frac{1}{\alpha} \cong \ln \left(\frac{M_{0}}{M_{X}}+\frac{M_{0}}{M_{X}}\right) \cong \ln \left(\frac{2 M_{0}}{M_{X}}\right)
$$

From experiments $1 / \alpha \cong 137.0359997$ and from the current observations[22,23,24], magnitude of the Hubble constant is, $H_{0} \cong 70.4_{-1.4}^{+1.3} \mathrm{Km} / \mathrm{sec} / \mathrm{Mpc}$. Thus

$$
\begin{aligned}
M_{X} & \cong e^{-\frac{1}{\alpha}}\left(\frac{c^{3}}{G H_{0}}\right) \cong e^{-\frac{1}{\alpha}} \cdot 2 M_{0} \\
& \cong(5.32 \text { to } 5.53) \times 10^{-7} \mathrm{Kg} .
\end{aligned}
$$

If $N \cong 6.022141793 \times 10^{23}$ is the Avogadro number and $m_{e}$ is the rest mass of electron, surprisingly it is noticed that, $N . m_{e} \cong 5.485799098 \times 10^{-7} \mathrm{Kg}$ and this is close to the above estimation of $M_{X}$. Thus it can be suggested that,

$$
\frac{M_{X}}{m_{e}} \cong N
$$

In this way, Avogadro number can be coupled with the cosmic, atomic and particle physics. Then with reference to $\left(N . m_{e}\right)$, the obtained cosmic Hubble mass is $M_{0} \cong 8.957532458 \times 10^{52} \mathrm{Kg}$ and thus the obtained Hubble's constant is $H_{0} \cong \frac{c^{3}}{2 G M_{0}} \cong 69.54 \mathrm{Km} / \mathrm{sec} / \mathrm{Mpc}$. Note that large dimensionless constants and compound physical constants reflect an intrinsic property of nature [25,26].
Whether to consider them or discard them depends on the physical interpretations, logics, experiments, observations and our choice of scientific interest. In most of the critical cases, 'time' only will decide the issue. The mystery can be resolved only with further research, analysis, discussions and encouragement.

Concept -7: For any observable charged particle, there exist two kinds of masses and their mass ratio is 295.0606339. Let this number be $\gamma$. First kind of mass seems to be the 'gravitational or observed' mass and the second kind of mass seems to be the 'electromagnetic' mass. This idea can be applied to proton and electron.

This number is obtained in the following way. In the Planck scale, similar to the Planck mass, with reference to the elementary charge, a new mass unit can be constructed in the following way.

$$
\begin{gathered}
M_{C} \cong \sqrt{\frac{e^{2}}{4 \pi \varepsilon_{0} G}} \cong 1.859210775 \times 10^{-9} \mathrm{Kg} \\
M_{C} c^{2} \cong \sqrt{\frac{e^{2} c^{4}}{4 \pi \varepsilon_{0} G}} \cong 1.042941 \times 10^{18} \mathrm{GeV}
\end{gathered}
$$

Here $e$ is the elementary charge. How to interpret this mass unit? Is it a primo rdial massive charged particle? If two such oppositely charged partic les annihilate, a large amount of energy can be released. This may be the root cause of cosmic energy reservoir. Such pairs may be the chief constituents of black holes. In certain time interval with a well defined quantum rules they annihilate and release a large amount of energy in the form of $\gamma$ photons. In the Hubble volume, with its pair annihilation, "orig in of the CMBR" can be understood. Clearly speaking, gravitational and electromagnetic force ratio of $M_{X}$ is $\gamma^{2}$.

$$
\frac{M_{X}}{M_{C}} \cong \sqrt{\frac{4 \pi \varepsilon_{0} G M_{X}^{2}}{e^{2}}} \cong 295.0606338=\gamma
$$

It can be interpreted that, if $5.486 \times 10^{-7} \mathrm{Kg}$ is the observable or gravitational mass of $M_{X}$, then $M_{C}$ is the electro magnetic mass of $M_{X}$. With reference to the electron rest mass,

$$
\left(\frac{M_{X}}{m_{e}}\right)^{2} \cong \gamma^{2} \cdot \frac{e^{2}}{4 \pi \varepsilon_{0} G m_{e}^{2}} \cong N^{2}
$$

Concept-8: If $\hbar$ is the quantum of the gravitational angular momentum, then the electromagnetic quantum can be expressed as $\left(\frac{\hbar}{\gamma}\right)$. Thus the ratio,

$$
\begin{gathered}
\left(\frac{\hbar}{\gamma}\right) \div\left(\frac{e^{2}}{4 \pi \varepsilon_{0} c}\right) \cong\left(X_{E} \alpha\right)^{-1} \cong \sin \theta_{W} \\
\cong 0.464433353
\end{gathered}
$$

where $\sin \theta_{W}$ is very close to the weak mixing angle

Concept-9: In modified quark SUSY[17,18], if $Q_{f}$ is the 
mass of quark fermion and $Q_{b}$ is the mass of quark boson, then

$$
\frac{Q_{f}}{Q_{b}} \cong \Psi \cong 2.2627062
$$

and $\left(1-\frac{1}{\Psi}\right) Q_{f}$ represents the effective quark fermion mass. The number $\Psi$ can be fitted with the following empirical relation

$$
\Psi^{2} \ln \left(1+\sin ^{2} \theta_{W}\right) \cong 1
$$

With this idea super symmetry can be observed in the low and high energy strong interactions and can also be observed in the electroweak interactions $[17,18]$.

\section{To Fit the Rest Masses of Electron, Proton and Neutron}

If $m_{e}$ is the light charged elementary particle and $M_{X}$ is the heavy charged elementary particle to be detected or observed, it is possible to represent the relation in the following form.

$$
\begin{gathered}
\sqrt{m_{e} M_{X}} \cong \gamma \sqrt{\frac{e^{2}}{4 \pi \varepsilon_{0} G_{A}}} \\
\text { let, } \sqrt{\frac{e^{2}}{4 \pi \varepsilon_{0} G_{A}} \cong m_{c}} \\
m_{e} \cong \frac{\left(\gamma m_{c}\right)^{2}}{M_{X}}
\end{gathered}
$$

In this way the origin of the electron rest mass can be understood. It is noticed that,

$$
\left(m_{e}^{2} \cdot \gamma m_{c}\right)^{\frac{1}{3}} c^{2} \cong 4695.8239 \mathrm{MeV}
$$

and is roughly 5 times greater than the nucleon rest energy.

If $k=\frac{G_{A} M_{X} m_{e}}{\hbar c} \cong 635.3132, k^{\frac{1}{4}} \cong 5.0205$.

An attempt is made to fit the rest masses of proton and neutron in the following way.

$$
\begin{gathered}
m_{p} c^{2} \cong\left(\frac{1}{k}\right)^{\frac{1}{4}}\left(m_{e}^{2} \cdot \gamma m_{c}\right)^{\frac{1}{3}} c^{2}+\ln \left(\frac{k}{2}\right) m_{e} c^{2} \\
\cong 938.2738 \mathrm{MeV} \\
m_{n} c^{2} \cong\left(\frac{1}{k}\right)^{\frac{1}{4}}\left(m_{e}^{2} \cdot \gamma m_{c}\right)^{\frac{1}{3}} c^{2}+\ln (2 \pi k) m_{e} c^{2} \\
\cong 939.56713 \mathrm{MeV} \\
\left(m_{n}-m_{p}\right) c^{2} \cong\left[\ln (2 \pi k)-\ln \left(\frac{k}{2}\right)\right] m_{e} c^{2} \\
\cong 1.29335 \mathrm{MeV}
\end{gathered}
$$

In support of these relations an attempt is made to implement the number $k$ in fitting the nuclear binding energy constants and other areas of physics like strong interaction range, potential energy of electron in hydrogen atom, electroweak physics etc.

\subsection{To Fit the Nuclear Binding Energy Constants}

The semi-empirical mass formula (SEMF) is used to approximate the mass and various other properties of an atomic nucleus[27,28,29]. As the name suggests, it is based partly on theory and partly on empirical measurements. Based on the 'least squares fit', volu me energy coefficient is $a_{v}=15.78 \mathrm{MeV}$, surface energy coefficient is $a_{s}=18.34$ $\mathrm{MeV}$, coulombic energy coefficient is $a_{c}=0.71 \mathrm{MeV}$, asymmetric energy coefficient is $a_{a}=23.21 \mathrm{MeV}$ and pairing energy coefficient is $a_{p}=12 \mathrm{MeV}$. The semi empirical mass formula is

$B E \cong A a_{v}-A^{\frac{2}{3}} a_{s}-\frac{Z(Z-1)}{A^{\frac{1}{3}}} a_{c}-\frac{(A-2 Z)^{2}}{A} a_{a} \pm \frac{1}{\sqrt{A}} a_{p}(22)$

It is noticed that,

$$
a_{v}+a_{s} \cong a_{a}+a_{p} \cong \frac{3}{2} a_{a} \cong \frac{m_{p} c^{2}}{1+\sqrt{k}} \cong 35.8045 \mathrm{MeV}(23)
$$

Asymmetric energy constant be

$$
a_{a} \cong \frac{2}{3} \cdot \frac{m_{p} c^{2}}{1+\sqrt{k}} \cong 23.870 \mathrm{MeV}
$$

Pairing energy constant be

$$
a_{p} \cong \frac{a_{a}}{2} \cong \frac{1}{3} \cdot \frac{m_{p} c^{2}}{1+\sqrt{k}} \cong 11.935 \mathrm{MeV}
$$

Let the maximum nuclear binding energy per nucleon be

$$
\left(B_{A}\right)_{\max } \cong \frac{1}{4} \cdot \frac{m_{p} c^{2}}{1+\sqrt{k}} \cong 8.9511 \mathrm{MeV}
$$

Coulo mbic energy constant be

$$
a_{c} \cong \sqrt{\alpha} \cdot\left(B_{A}\right)_{\max } \cong 0.7647 \mathrm{MeV}
$$

Table 1. SEMF binding energy with the proposed energy coefficients

\begin{tabular}{|c|c|c|c|}
\hline$Z$ & $A$ & $(B E)_{c a l}$ in $\mathrm{MeV}$ & $(B E)_{\text {meas }}$ in $\mathrm{MeV}$ \\
\hline 26 & 56 & 492.18 & 492.254 \\
\hline 28 & 62 & 546.67 & 545.259 \\
\hline 34 & 84 & 727.78 & 727.341 \\
\hline 50 & 118 & 1007.80 & 1004.950 \\
\hline 60 & 142 & 1184.55 & 1185.145 \\
\hline 79 & 197 & 1556.72 & 1559.40 \\
\hline 82 & 208 & 1627.20 & 1636.44 \\
\hline 92 & 238 & 1805.71 & 1801.693 \\
\hline
\end{tabular}

Surface energy constant be

$$
a_{s} \cong 2\left(B_{A}\right)_{\max }\left(1+\sqrt{\frac{a_{c}}{a_{a}}}\right) \cong 19.504 \mathrm{MeV}
$$

Volume energy constant be

$$
a_{v} \cong 2\left(B_{A}\right)_{\max }\left(1-\sqrt{\frac{a_{c}}{a_{a}}}\right) \cong 16.30 \mathrm{MeV}
$$


In table-1 within the range of $(Z=26 ; A=56)$ to $(Z=92 ; A=238)$ nuclear binding energy is calculated and compared with the measured binding energy[30]. Colu mn-3 represents the calculated binding energy and column-4 represents the measured binding energy.

\subsection{Proton-nucleon Stability Relation}

It is noticed that

$$
\frac{A_{s}}{2 Z} \cong 1+2 Z\left(\frac{a_{c}}{a_{s}}\right)^{2}
$$

where $A_{s}$ is the stable mass number of $Z$. This is a direct relation. Assuming the proton number $Z$, in general, for all atoms, lower stability can be fitted directly with the following relation[27].

$$
A_{s} \cong 2 Z\left[1+2 Z\left(\frac{a_{c}}{a_{s}}\right)^{2}\right] \cong 2 Z+Z^{2} * 0.00615
$$

if $Z=21, \quad A_{s} \cong 44.71 ; \quad$ if $Z=29, \quad A_{s} \cong 63.17 ; \quad$ if $Z=47, \quad A_{s} \cong 107.58$; if $Z=53, A_{s} \cong 123.27$ if $Z=60$, $A_{s} \cong 142.13 ; \quad$ if $\quad Z=79, \quad A_{s} \cong 196.37 ; \quad$ if $\quad Z=83$, $A_{s} \cong 208.35 ;$ if $Z=92, A_{s} \cong 236.03$;

Stable super heavy elements can be predicted with this relation. In between $Z=30$ to $Z=60$ obtained $A_{s}$ is lower compared to the actual $A_{s}$. It is noticed that, upper stability in light and medium atoms up to $Z \approx 56$ can be fitted with the following relation.

$$
\begin{gathered}
A_{s} \cong 2 Z\left[1+2 Z\left(\left(\frac{a_{c}}{a_{s}}\right)^{2}+\left(\frac{a_{c}}{4\left(B_{A}\right)_{\max }}\right)^{2}\right)\right] \\
\cong 2 Z+Z^{2} * 0.0080
\end{gathered}
$$

From this relation for $Z=56$, obtained upper $A_{s} \cong 137.1$. Note that, for $Z=56$, actual stable $A_{s} \cong 137 \cong \frac{1}{\alpha}$ where $\alpha$ is the fine structure ratio. This seems to be a nice and interesting coincidence. In between 0.00615 and 0.0080 , for light and medium atoms up to $Z \approx 56$ or $A_{s} \approx 137$, mean stability can be fitted with the following relation.

$$
A_{s} \cong 2 Z+Z^{2} * 0.00706
$$

Surprisingly it is noticed that, in this relation, $0.0071 \approx \alpha$. Thus up to $Z \cong 56$ or $A_{s} \approx 137$, mean stability can be expressed as

$$
A_{s} \approx 2 Z+\left(Z^{2} \alpha\right)
$$

\section{To Fit the Rms Radius of Proton}

Let $R_{p}$ be the rms radius of proton. Define two radii $R_{1}$ and $R_{2}$ as follows.

$$
\begin{aligned}
R_{1} & \cong\left(\frac{\hbar c}{G_{A} m_{p}^{2}}\right)^{2} \frac{2 G_{C} m_{p}}{c^{2}} \cong 1.9637 \times 10^{-25} \mathrm{~m} \\
R_{2} & \cong\left(\frac{\hbar c}{G_{A} m_{p}^{2}}\right)^{3} \frac{2 G_{C} m_{p}}{c^{2}} \cong 5.521 \times 10^{-11} \mathrm{~m}
\end{aligned}
$$

It is noticed that,

$$
R_{p} \cong\left(R_{1} R_{2}^{2}\right)^{\frac{1}{3}} \cong 8.4278 \times 10^{-16} \mathrm{~m}
$$

Thus,

$$
R_{p} \cong\left(\frac{\hbar c}{G_{A} m_{p}^{2}}\right)^{8 / 3} \frac{2 G_{C} m_{p}}{c^{2}}
$$

This can be compared with the 2010 CODATA recommended rms radius of proton $0.8775(51) \mathrm{fm}$. Recent work on the spectrum of muonic hydrogen (an exotic atom consisting of a proton and a negative muon) indicates a significantly lower value for the proton charge radius, $R_{p} \cong 0.84184(67) \mathrm{fm}$ and the reason for this discrepancy is not clear. Th is is 10 times more precise than all the previous determinations $[31,32]$. Thus from proton rest mass and rms radius,

$$
\begin{aligned}
& G_{A} \cong\left(\frac{2 G_{C} m_{p}}{R_{p} c^{2}}\right)^{\frac{3}{8}}\left(\frac{\hbar c}{m_{p}^{2}}\right) \\
& N \cong\left(\frac{2 G_{C} m_{p}}{R_{p} c^{2}}\right)^{\frac{3}{8}}\left(\frac{\hbar c}{G_{C} m_{p}^{2}}\right)
\end{aligned}
$$

Here the most interesting thing is that, $R_{2}$ is very close to the Bohr radius of Hydrogen atom. It is very interesting to note that, with $R_{2}$ ionic radii of atoms can be fitted very easily as

$$
(R)_{A} \cong A^{1 / 3} \cdot\left(\frac{R_{2}}{\sqrt{2}}\right) \cong A^{1 / 3} \times 3.904 \times 10^{-11} \mathrm{~m}
$$

where $(R)_{A}$ is the ionic radius of mass number $A$. If $A=7,(R)_{A} \cong 0.0747 \mathrm{~nm}$, if $A=23,(R)_{A} \cong 0.111 \mathrm{~nm}$ and if $A=39,(R)_{A} \cong 0.132 \quad \mathrm{~nm}$. Their corresponding recommended radii are $0.076 \mathrm{~nm}, 0.102 \mathrm{~nm}$ and $0.138 \mathrm{~nm}$ respectively[31,32].

\section{To Fit the Characteristic Potential Radius of Nucleus $1.4 \mathrm{fm}$}

It is noticed that, gram mole is a black hole where the operating gravitational constant is $\left(G_{A}\right)$ but not $\left(G_{C}\right)$. That means for the simplest case of gram mole of electrons or gram mole of protons, there exist $N$ number of electrons or $N$ number of protons. Let it follows the concept of Schwarzschild radius. It can be expressed in the following way. Let us define two radii $R_{3}$ and $R_{4}$ as follows. 


$$
\begin{gathered}
R_{3} \cong \frac{2 G_{A}\left(N m_{e}\right)}{c^{2}} \cong 4.9066 \times 10^{-10} \mathrm{~m} \\
R_{4} \cong \frac{2 G_{A}\left(N m_{p}\right)}{c^{2}} \cong 9.009 \times 10^{-7} \mathrm{~m} \\
V_{3} \cong \frac{4 \pi}{3} R_{3}^{3} \\
V_{4} \cong \frac{4 \pi}{3} R_{4}^{3}
\end{gathered}
$$

For the above two cases, the characteristic mean distance $(\lambda)$ in between $N$ electrons or in between $N$ protons, can be obtained as

$$
\begin{aligned}
& \lambda_{3} \cong\left(\frac{V_{3}}{N}\right)^{\frac{1}{3}} \\
& \lambda_{4} \cong\left(\frac{V_{4}}{N}\right)^{\frac{1}{3}}
\end{aligned}
$$

It is noticed that,

$$
\lambda_{34} \cong\left(\lambda_{3} \lambda_{4}^{2}\right)^{\frac{1}{3}} \cong 1.4 \times 10^{-15} \mathrm{~m}
$$

This can be compared with the characteristic alpha scattering experimental radius [31] of nucleus $\approx 1.4 \mathrm{fm}$. Based on the Yukawa's Pion exchange model nuclear interaction range is $1.4 \mathrm{fm}$. Thus if $m_{\pi}^{ \pm}$is the charged pion rest mass,

$$
N \cong\left(\frac{3}{32 \pi}\right)^{\frac{1}{5}}\left(\frac{\hbar c}{G_{C}\left(m_{p}^{2} m_{e}\right)^{1 / 3} m_{\pi}^{ \pm}}\right)^{3 / 5}
$$

\section{Applications of the Proposed Assumptions and Concepts}

\subsection{PART-1 : Applications in Particle and Nuclear Physics}

\subsubsection{Application-1: The Characteristic Nuclear Charge Radius}

If $H_{0} \cong 69.54 \mathrm{Km} / \mathrm{sec} / \mathrm{Mpc}, R_{s}$ is the characteristic radius of nucleus, it is noticed that,

$$
R_{s} \cong\left(\frac{m_{p}}{M_{X}}\right)^{2} \frac{c}{H_{0}} \cong 1.2368 \times 10^{-15} \mathrm{~m}
$$

where $m_{p}$ is the proton rest mass. This can be compared with the characteristic charge radius of the nucleus and the strong interaction range.

\subsubsection{Application-2: Scattering Distance between Electron and the Nucleus}

If $R_{s} \cong 1.21$ to $1.22 \mathrm{fm}$ is the minimum scattering distance between electron and the nucleus, it is noticed that,

$$
R_{s} \cong\left(\frac{\hbar c}{G_{A} m_{e}^{2}}\right)^{2} \cdot \frac{2 G_{C} m_{e}}{c^{2}} \cong 1.21565 \times 10^{-15} \mathrm{~m}
$$

Here $M_{X}$ is the molar electron mass. Here it is very interesting to consider the role of the Schwarzs child radius of the 'electron mass'.

\subsubsection{Aplication-3: To Fit the Charged Lepton Rest Masses}

Muon and tau rest masses can be fitted in the following way[33]. Let $R_{s}$ be the characteristic nuclear unit size. The key relation seems to be

$$
\left(\frac{\hbar c}{G_{A} m_{e}^{2}}\right)^{2} \cong \frac{R_{s} c^{2}}{2 G_{C} m_{e}}
$$

Considering the ratio of the volumes $\frac{4 \pi}{3} R_{s}^{3}$ and $\frac{4 \pi}{3}\left(\frac{2 G_{C} m_{e}}{c^{2}}\right)^{3}$, let

$$
\ln \left(\frac{R_{s} c^{2}}{2 G_{C} m_{e}}\right)^{3} \cong 289.805 \approx \gamma
$$

Now muon and tau masses can be fitted with the following relation $[17,18]$.

$$
\left(m_{l} c^{2}\right)_{x} \cong\left[\gamma^{3}+\left(x^{2} \gamma\right)^{x} \sqrt{N}\right]^{\frac{1}{3}} \cdot \frac{m_{e} c^{2}}{\gamma}
$$

where $\mathrm{x}=0,1$ and 2 . At $\mathrm{x}=0,\left(m_{l} c^{2}\right)_{0} \cong m_{e} c^{2}$. At $\mathrm{x}=1$, $\left(m_{l} c^{2}\right)_{1} \cong 105.9 \mathrm{MeV}$ and can be compared with the rest mass of muon (105.66 MeV). At $\mathrm{x}=2,\left(m_{l} c^{2}\right)_{2} \cong 1777.4$ $\mathrm{MeV}$ and can be compared with the rest mass of tau (1777.0 $\mathrm{MeV}) . \mathrm{x}=0,1$ and 2 can be considered as the 3 characteristic vibrating modes.

\subsubsection{Aplication-4: Electromagnetic and Strong Interaction Ranges}

For electron, starting from $\left(c / H_{0}\right)$, its characteristic interaction ending range can be expressed as

$$
r_{e e} \cong \frac{e^{2}}{4 \pi \varepsilon_{0}\left(m_{e} / \gamma\right) c^{2}} \cong \gamma \frac{e^{2}}{4 \pi \varepsilon_{0} m_{e} c^{2}} \cong 8.315 \times 10^{-13} \mathrm{~m}(56)
$$

Similarly, for proton, its characteristic interaction starting range can be expressed as

$r_{s s} \cong \frac{e^{2}}{4 \pi \varepsilon_{0}\left(m_{p} / \gamma\right) c^{2}} \cong \gamma \frac{e^{2}}{4 \pi \varepsilon_{0} m_{p} c^{2}} \cong 4.53 \times 10^{-16} \mathrm{~m}(57)$

\subsubsection{Application-5: Ratio of Electro magnetic and Strong Interaction Ending Range}

Ratio of electromagnetic ending interaction range and strong interaction ending range can be expressed as 


$$
\frac{r_{e e}}{r_{s e}} \cong \frac{G_{A} M_{X} m_{e}}{\hbar c} \cong k \cong 635.3131866
$$

Thus if $r_{e e} \cong 8.315 \times 10^{-13} \mathrm{~m}, r_{s e} \cong 1.309 \times 10^{-15} \mathrm{~m}$,

$$
\left(\frac{r_{e e}}{r_{s e}}\right)^{2} \cong\left(\frac{G_{A} M_{X} m_{e}}{\hbar c}\right)^{2} \cong k^{2}
$$

Interesting observation is

$$
\frac{r_{s s}+r_{s e}}{2} \cong 0.881 \times 10^{-15} \mathrm{~m}
$$

This can be considered as the mean strong interaction range and is close to the proton rms radius!

\subsubsection{Application-6}

For any elementary particle of charge $e$, electro magnetic mass $(m / \gamma)$ and characteristic radius $R$, it can be assumed as

$$
\frac{e^{2}}{4 \pi \varepsilon_{0} R} \cong \frac{1}{2}\left(\frac{m}{\gamma}\right) c^{2}
$$

This idea can be applied to proton as well as electron. Electron's characteristic radius is

$$
R_{e} \cong 2 \gamma \frac{e^{2}}{4 \pi \varepsilon_{0} m_{e} c^{2}} \cong 1.663 \times 10^{-12} \mathrm{~m}
$$

Similarly proton's characteristic radius is

$$
R_{p} \cong 2 \gamma \frac{e^{2}}{4 \pi \varepsilon_{0} m_{p} c^{2}} \cong 0.906 \times 10^{-15} \mathrm{~m}
$$

\subsubsection{Application-7: Potential Energy of Electron in Hydrogen Atom}

Let $E_{p}$ be the potential energy of electron in the Hydrogen atom. It is noticed that,

$$
E_{p} \cong \frac{e^{2}}{4 \pi \varepsilon_{0} a_{0}} \cong\left(\frac{\hbar c}{G_{A} M_{X} m_{e}}\right) \frac{(\hbar / \gamma) c}{\sqrt{R_{e} R_{p}}} \cong 27.12493044 \mathrm{eV} \text { (64) }
$$

where $a_{0}$ is the Bohr radius[34,35]. With $99.6822 \%$ this is matching with $\alpha^{2} m_{e} c^{2} \cong 27.21138388 \quad \mathrm{eV}$. After simplification it takes the following form.

$$
E_{p} \cong\left(\frac{\hbar c}{G_{A} M_{X} m_{e}}\right)^{2} \frac{\sqrt{m_{p} m_{e}} c^{2}}{2} \cong \alpha^{2} m_{e} c^{2}
$$

Thus the Bohr radius can be expressed as

$$
a_{0} \cong\left(\frac{G_{A} M_{X} m_{e}}{\hbar c}\right)^{2} \frac{2 e^{2}}{4 \pi \varepsilon_{0} \sqrt{m_{p} m_{e}} c^{2}}
$$

Electron's $n^{\text {th }}$ orbit radii can be expressed as

$$
a_{n} \cong\left(\frac{G_{A} M_{X} m_{e}}{\hbar c}\right)^{2} \frac{2(n e)^{2}}{4 \pi \varepsilon_{0} \sqrt{m_{p} m_{e}} c^{2}} \cong n^{2} \cdot a_{0}
$$

where $a_{n}$ is the radius of $n^{\text {th }}$ orbit and $n=1,2,3, .$. Thus in Hydrogen atom, potential energy of electron in $n^{\text {th }}$ orbit can be expressed as

$$
\frac{e^{2}}{4 \pi \varepsilon_{0} a_{n}} \cong\left(\frac{\hbar c}{G_{A} M_{X} m_{e}}\right)^{2} \frac{\sqrt{m_{p} m_{e}} c^{2}}{2 n^{2}}
$$

Note that, from the atomic theory it is well established that, total number of electrons in a shell of principal quantum number $n$ is $2 n^{2}$. Thus on comparison, it can suggested that, $\left(\frac{\hbar c}{G_{A} M_{X} m_{e}}\right)^{2} \sqrt{m_{p} m_{e}} c^{2}$ is the potential energy of $2 n^{2}$ electrons and potential energy of one electron is equal to $\left(\frac{\hbar c}{G_{A} M_{X} m_{e}}\right)^{2} \frac{\sqrt{m_{p} m_{e}} c^{2}}{2 n^{2}}$.

\subsubsection{Application-8: Magnetic Moments of the Nucleon}

If $\left(\alpha X_{E}\right)^{-1} \cong \sin \theta_{W}$, magnetic moment of electron can be expressed as[36,37]

$$
\mu_{e} \cong \frac{1}{2} \sin \theta_{W} \cdot e c \cdot r_{e e} \cong 9.274 \times 10^{-24} \mathrm{~J} / \text { tesla }
$$

It can be suggested that electron's magnetic moment is due to the electromagnetic interaction range. Similarly magnetic moment of proton is due to the strong interaction ending range.

$$
\mu_{p} \cong \frac{1}{2} \sin \theta_{W} \cdot e c \cdot r_{s e} \cong 1.46 \times 10^{-26} \mathrm{~J} / \text { tesla }
$$

If proton and neutron are the two quantum states of the nucleon, by considering the mean strong interaction range $\left(\frac{r_{s s}+r_{s e}}{2}\right)$, magnetic moment of neutron can be fitted as

$$
\mu_{n} \cong \frac{1}{2} \sin \theta_{W} \cdot e c \cdot\left(\frac{r_{s s}+r_{s e}}{2}\right) \cong 9.82 \times 10^{-27} \mathrm{~J} / \text { tesla }
$$

\subsubsection{Application-9: To Corre late the Charged Higgs} Fermion Mass and the Electron Mass

If $M_{H f}$ is the charged Higgs fermion, it is noticed that,

$$
\frac{M_{H f}}{m_{e}} \cong \frac{m_{e} c^{2}}{F_{W} R_{s}}
$$

Thus,

$$
m_{e} c^{2} \cong \sqrt{M_{H f} c^{2} \cdot F_{W} R_{s}}
$$

From relation (52),

$$
M_{H f} c^{2} \cong \frac{1}{2}\left(\frac{G_{A} M_{X} m_{e}}{\hbar c}\right)^{2} m_{e} c^{2} \cong 103125.417 \mathrm{MeV}
$$

If Higgs fermion and Higgs boson mass ratio is 2.2627, then obtained Higgs boson mass is $45576.27 \mathrm{MeV}$ and the most surprising thing is that, Higgs boson pair generates the neutral $Z$ boson of rest energy $91152.53 \mathrm{MeV}$. Estimated top quark rest energy[17,18] is $182160 \mathrm{MeV}$ and its corresponding boson is $80505.6 \mathrm{MeV}$. Thus the surprising thing is that, susy boson of the top quark seems to be the electroweak $\mathrm{W}$ boson. Another interesting idea is that $\mathrm{W}$ 
boson and Higgs boson generate a neutral boson of mass 126 $\mathrm{GeV}$. It can be suggested that, $\mathrm{W}$ boson pair generates a neutral boson of rest energy $161 \mathrm{GeV}$.

\subsection{PART-2 : Applications in Cos mology}

\subsubsection{Application-10: To fit the Hubble's Constant}

Combining the relations (51) and (52) and if $H_{0} \cong 69.54$ $\mathrm{Km} / \mathrm{sec} / \mathrm{Mpc}$, it is noticed that,

$$
\frac{\hbar c}{G m_{p} \sqrt{M_{0} m_{e}}} \cong 0.991415
$$

Surprisingly this ratio is close to unity! How to interpret this ratio? From this relation it can be suggested that, along with the cosmic variable, $H_{0}$, in the presently believed atomic and nuclear physical constants, on the cosmological time scale, there exists one variable physical quantity. 'Rate of change' in its magnitude may be a measure of the present cosmic acceleration. Thus independent of the cosmic red shift and CMBR observations, fro $m$ the atomic and nuclear physics, cosmic acceleration can be verified. Based on the above coincidence, magnitude of the present Hubble's constant can be expressed as

$$
H_{0} \cong \frac{G m_{p}^{2} m_{e} c}{2 \hbar^{2}} \cong 70.75 \mathrm{Km} / \mathrm{sec} / \mathrm{Mpc}
$$

\subsubsection{Application-11: Pair Creation of $\mathrm{M}_{\mathrm{c}}$ within the Hubble} Volume and the CMBR Temperature

Pair particles creation and annihilation is a characteristic phenomena in 'free space', and is the basic idea of quantum fluctuations of the vacuum. In the expanding universe, from relation (8) by considering the proposed charged $M_{C}$ and its pair annihilation as characteristic cosmic phenomena, origin of the is otropic $\mathrm{CMB}$ radiation can be addressed. At any time $t$, it can be suggested that

$$
k_{B} T_{t} \cong \sqrt{\frac{M_{C}}{M_{t}}} \cdot 2 M_{C} c^{2}
$$

where $M_{t}$ is the cosmic mass at time $t$. Please note that, at present

$$
T_{t} \cong \sqrt{\frac{M_{C}}{M_{0}}} \cdot \frac{2 M_{C} c^{2}}{k_{B}} \cong 3.52{ }^{0} \mathrm{~K}
$$

Qualitatively and quantitatively this can be compared with the present CMBR temperature $2.725{ }^{0} \mathrm{~K}$. But it has to be discussed in depth. It seems to be a direct consequence of the Mach's principle.

\subsubsection{Application-12: A Quantitative Approach to Understand the CMBR Radiation}

It is noticed that, there exists a very simple relation in between the cosmic critical density, matter density and the thermal energy density. It can be expressed in the following way. At any time $t$,

$$
\left(\frac{\rho_{c}}{\rho_{m}}\right)_{t} \cong\left(\frac{\rho_{m}}{\rho_{T}}\right)_{t} \cong 1+\ln \left(\frac{M_{t}}{M_{C}}\right)
$$

where $\rho_{c} \cong M_{t}\left[\frac{4 \pi}{3}\left(\frac{c}{H_{t}}\right)^{3}\right]^{-1} \cong \frac{3 H_{t}^{2}}{8 \pi G}, \quad \rho_{m}$ is the matter density and $\rho_{T}$ is the thermal energy density expressed in gram $/ \mathrm{cm}^{3}$ or $\mathrm{Kg} / \mathrm{m}^{3}$. Considering the Planck - Coulo mb scale, at the beginning if $M_{t} \cong M_{C}$

$$
\begin{gathered}
\left(\frac{\rho_{c}}{\rho_{m}}\right)_{C} \cong\left(\frac{\rho_{m}}{\rho_{T}}\right)_{C} \cong 1 \\
\left(\rho_{c}\right)_{C} \cong\left(\rho_{m}\right)_{C} \cong\left(\rho_{T}\right)_{C}
\end{gathered}
$$

Thus at any time $t$,

$$
\begin{gathered}
\rho_{m} \cong \sqrt{\rho_{c} \cdot \rho_{T}} \\
\rho_{m} \cong\left[1+\ln \left(\frac{M_{t}}{M_{C}}\right)\right]^{-1} \rho_{c} \\
\rho_{T} \cong\left[1+\ln \left(\frac{M_{t}}{M_{C}}\right)\right]^{-2} \rho_{c} \cong\left[1+\ln \left(\frac{M_{t}}{M_{C}}\right)\right]^{-1} \rho_{m}
\end{gathered}
$$

In this way, observed matter density and the thermal energy density can be studied in a unified manner. The observed CMB anisotropy can be related with the inter galactic matter density fluctuations.

\subsection{Present Matter Density of the Universe}

From (76) at present if $H_{0} \cong 70.75 \mathrm{Km} / \mathrm{sec} / \mathrm{Mpc}$,

$$
\left(\rho_{m}\right)_{0} \cong\left[1+\ln \left(\frac{M_{0}}{M_{C}}\right)\right]^{-1}\left(\rho_{c}\right)_{0}
$$

$\cong 6.573 \times 10^{-32} \mathrm{gram} / \mathrm{cm}^{3}$ where $\left(\rho_{c}\right)_{0} \cong 9.4 \times 10^{-30} \mathrm{gram} / \mathrm{cm}^{3}$ and $\left[1+\ln \left(\frac{M_{0}}{M_{C}}\right)\right] \cong 143.013$. Based on the average mass-to-light ratio for any galaxy[6]

$$
\left(\rho_{m}\right)_{0} \cong 1.5 \times 10^{-32} \eta h_{0} \mathrm{gram} / \mathrm{cm}^{3}
$$

where for any galaxy, $\left\langle\frac{M_{G}}{L_{G}}\right\rangle \cong \eta\left(\frac{M_{\odot}}{L_{\odot}}\right)$ and the number $h_{0} \cong \frac{H_{0}}{100 \mathrm{Km} / \mathrm{sec} / \mathrm{Mpc}} \cong \frac{70.75}{100} \cong 0.7075$.

Note that elliptical galaxies probably comprise about $60 \%$ of the galaxies in the universe and spiral galaxies thought to make up about $20 \%$ percent of the galaxies in the universe. Almost $80 \%$ of the galaxies are in the form of elliptical and spiral galaxies. For spiral galaxies, $\eta h_{0}^{-1} \cong 9 \pm 1$ and for elliptical galaxies, $\eta h_{0}^{-1} \cong 10 \pm 2$. For our galaxy inner part, $\eta h_{0}^{-1} \cong 6 \pm 2$. Thus the average $\eta h_{0}^{-1}$ is very close to 8 to 9 and its corresponding matter density is close to (6.0 to 6.76$) \times 10^{-32} \mathrm{gram} / \mathrm{cm}^{3}$ and can be compared with the above proposed magnitude of $6.573 \times 10^{-32} \mathrm{gram} / \mathrm{cm}^{3}$.

\subsection{Present Ther mal Energy Density of the Uni verse}


At present if $H_{0} \cong 70.75 \mathrm{Km} / \mathrm{sec} / \mathrm{Mpc}$,

$$
\left(\rho_{T}\right)_{0} \cong\left[1+\ln \left(\frac{M_{0}}{M_{C}}\right)\right]^{-2}\left(\rho_{c}\right)_{0} \cong 4.6 \times 10^{-34} \mathrm{gram} / \mathrm{cm}^{3}(87)
$$

and thus

$$
\left(\rho_{T} c^{2}\right)_{0} \cong\left[1+\ln \left(\frac{M_{0}}{M_{C}}\right)\right]^{-2}\left(\rho_{c} c^{2}\right)_{0} \cong 4.131 \times 10^{-14} \mathrm{~J} / \mathrm{m}^{3}(88)
$$

At present if

$$
\left(\rho_{T} c^{2}\right)_{0} \cong a T_{0}^{4}
$$

where $a \cong 7.56576 \times 10^{-16} \mathrm{~J} / \mathrm{m}^{3} \mathrm{~K}^{4}$ is the radiation energy density constant, then obtained CMBR temperature is, $T_{0} \cong 2.718{ }^{0}$ Kelvin. This is accurately fitting with the observed CMBR temperature[24], $T_{0} \cong 2.725{ }^{0}$ Kelvin. Thus in this way, the present value of the Hubble's constant and the present CMBR temperature can be co-related with the following trial-error relation.

$$
\left[1+\ln \left(\frac{c^{3}}{2 G H_{0} M_{C}}\right)\right]^{-1} H_{0} \cong \sqrt{\frac{8 \pi G a T_{0}^{4}}{3 c^{2}}}
$$

\section{Discussion \& Conclusions}

String theory or QCD is not in a position to address the basics of cosmic structure[38]. In understanding the basic concepts of unification or theory of every thing, role of dark energy and dark matter is insignificant. Even though string theory was introduced for understanding the basics of strong interaction, its success seems to be a dile mma because of its higher dimensions and the non-coupling of the nuclear and Planck scale. Based on the proposed relations and applications, Hubble volume or Hubble mass, can be considered as a key tool in unification as well as cosmology. From relations $(51,52,75)$, if it is possible to identify the atomic cosmo logical physical variable, then by observing the rate of change in its magnitude (on the cosmological time scale), the "future" cosmic acceleration can be verified and thus the cosmic geometry can be confirmed from atomic and nuclear physics! Without the advancement of nanotechnology or fermi-technology this may not be possible. Not only that, independent of the cosmic red shift and CMBR observations "future" cosmic acceleration can be checked in this new direction.

Considering the proposed relations and concepts it is possible to say that there exists a strong relation between cosmic Hubble mass, Avogadro number and unification..

\section{ACKNOWLEDGEMENTS}

The first author is indebted to professor K. V. Krishna Murthy, Chairman, Institute of Scientific Research on Vedas (I-SERVE), Hyderabad, India and Shri K. V. R. S. Murthy, former scientist IICT (CSIR) Govt. of India, Director,
Research and Development, I-SERVE, for their valuable guidance and great support in developing this subject.

\section{REFERENCES}

[1] Sciama, D. W. "Modern Cosmology". Cambridge University Press. (1971) OCLC 6931707

[2] Hawking. S.W and George Francis Rayner Ellis. "The Large Scale Structure of Space-Time". Cambridge University Press. (1973)

[3] Raine, D. J. "Mach's Principle in general relativity". Royal Astronomical Society 171: 507. 1975.

[4] Bondi, et al. "The Lense-Thirring Effect and Mach's Principle". Physics Letters A. Vol-228, Issue 3, 7 April 1997, Pages 121-126.

[5] R. G. Vishwakarma. "A Machian Model of Dark Energy". Class.Quant.Grav. 19 (2002) 4747-4752.

[6] J. V. Narlikar. "An Introduction to Cosmology". 3rd edition, Cambridge University Press.(2002)

[7] U. V. S. Seshavatharam and S. Lakshminarayana.. Quantum Mechanics, Cosmic Acceleration and CMB Radiation. Global Journal of Science Frontier Research (A) Vol. 12 Issue 4, p.17, (2012).

[8] U. V. S. Seshavatharam and S. Lakshminarayana.. Atom, universe and the fundamental interactions. Global Journal of Science Frontier Research (A) Vol. 12 Issue 5, p.1, (2012).

[9] U. V. S. Seshavatharam and S. Lakshminarayana.. Is strong interaction - a cosmological manifestation? Global Journal of Science Frontier Research (A) Vol. 12 Issue 6, p.37, (2012).

[10] U. V. S. Seshavatharam and S. Lakshminarayana. Past, present and future of the Avogadro number. Global Journal of Science Frontier Research (A) Vol. 12 Issue 7, p.27, (2012).

[11] Abdus Salam. "Strong Interactions, Gravitation and Cosmology". Publ. in: NATO Advanced Study Institute, Erice, June16-July 6, 1972.

[12] P. Caldirola, M. Pavsic and Recami E. "Explaining the Large Numbers by a Hierarchy of Universes: A Unified Theory of Strong and Gravitational Interactions". IL Nuovo Cimento Vol. 48 B, No. 2, 11 Dec 1978.

[13] Abdus Salam. "Strong Interactions, Gravitation and Cosmology". Publ. in: NATO Advanced Study Institute, Erice, June16-July 6, 1972 .

[14] Salam A, Sivaram C. "Strong Gravity Approach to QCD and Confinement”. Mod. Phys. Lett., 1993, v. A8(4), 321-326.

[15] Recami E. "Elementary Particles as Micro-Universes, and "Strong Black-holes": A Bi-Scale Approach to Gravitational and Strong Interactions". Preprint NSF-ITP-02-04, posted in the arXives as the e-print physics/0505149, and references therein.

[16] Seshavatharam. U.V.S. The primordial cosmic black hole and the cosmic axis of evil. International journal of astronomy. 1(2): 20-37. 2012. 
[17] U. V. S. Seshavatharam and S. Lakshminarayana.. Super Symmetry in Strong and Weak interactions. Int. J. Mod. Phys. E, Vol.19, No.2, (2010), p.263

[18] U. V. S. Seshavatharam and S. Lakshminarayana.. SUSY and strong nuclear gravity in (120-160) $\mathrm{GeV}$ mass range. Hadronic journal, Vol-34, No 3, 2011 June, p.277

[19] U. V. S. Seshavatharam and S. Lakshminarayana.. Strong nuclear gravity - a brief report. Hadronic journal, Vol-34, No 4, 2011 Aug.p.431

[20] U. V. S. Seshavatharam and S. Lakshminarayana.. Nucleus in Strong nuclear gravity. Proceedings of the DAE Symp. on Nucl. Phys. 56 (2011) p.302.

[21] U. V. S. Seshavatharam and S. Lakshminarayana. Integral charge SUSY in Strong nuclear gravity. Proceedings of the DAE Symp. on Nucl. Phys. 56 (2011) p.842.

[22] J. Huchara. "Estimates of the Hubble Constant", 2009. Harvard-Smithsonian Center for Astrophysics. http://hubble. plot.dat

[23] W. L. Freedman et al. "Final Results from the Hubble Space Telescope Key Project to Measure the Hubble Constant". The Astrophysical Journal 553 (1): 47-72.2001.

[24] N. Jarosik et al. "Seven-Year Wilson Microwave Anisotropy Probe (WMAP) Observations: Sky Maps, Sy stematic Errors, and Basic Results" (PDF). nasa.gov. Retrieved 2010-12-02.

[25] P. A. M. Dirac. "The cosmological constants". Nature, 139, 323,1937

[26] P. A. M. Dirac. "A new basis for cosmology". Proc. Roy. Soc. A $165,199,1938$.

[27] P. Roy Chowdhury et al. Modified Bethe-Weizsacker mass formula with isotonic shift and new drip lines. Mod. Phys. Lett. A20 (2005) p.1605-1618
[28] W. D. Myers et al. Table of Nuclear Masses according to the 1994 Thomas-Fermi Model.(from nsdssd.lbl.gov)

[29] U. V. S. Seshavatharam and S. Lakshminarayana. Role of the fine structure ratio in fitting the SEMF energy constants and super heavy magic nu mbers. Hadronic journal. Vol. 35, No. 1, p.113, 2012.

[30] G. Audi and A.H. Wapstra. The 1993 atomic mass evolution.(I) Atomic mass table. Nuclear physics, A 565, 1993, p 1-65.

[31] Geiger H and Marsden E. "On a diffuse reaction of the particles”. Proc. Roy. Soc., Ser. A 82: 495-500, 1909.

[32] Michael O. Distler et al. The RMS Charge Radius of the Proton and Zemach Moments. Phys. Lett. B696:343-347, 2011

[33] Particle Data Group (W.-M. Yao et al.), J. Phys. G 33 (2006) 1, http://pdg.bbb.gov.

[34] N. Bohr. "On the Constitution of Atoms and Molecules". (Part-1) Philos. Mag. 26, 11913

[35] N. Bohr. "On the Constitution of Atoms and Molecules". (Part-2, Systems containing only a Single Nucleus). Philos. Mag. 26, 476, 1913

[36] P.A.M.Dirac. The quantum theory of electron. Proc. Roy. Soc. A $117,610,1928$.

[37] U. V. S. Seshavatharam and S. Lakshminarayana.. Atomic gravitational constant and the origin of elementary magnetic moments. Hadronic journal, Vol-33, No 6, 2010 Dec, p.655

[38] David Gross, "Einstein and the search for Unification". Current science, Vol. 89, No. 12, 25 Dec 2005. 